\title{
Parvovirus B19 Infection in Pregnancy: Implications for Childhood Outcomes?
}

\author{
Amish Jain ${ }^{1}$, Edmond Kelly ${ }^{1,2}$ and Vibhuti Shah ${ }^{*}, 1,2$ \\ ${ }^{I}$ Department of Paediatrics, Mount Sinai Hospital, University of Toronto, Canada \\ ${ }^{2}$ Faculty of Medicine, University of Toronto, Mount Sinai Hospital, Canada
}

\begin{abstract}
Background: Maternal parvovirus B19 infection can lead to serious consequences in the fetus including fetal death, non-immune hydrops fetalis and possibly long-term adverse neurodevelopmental outcome. To date, there has been no systematic review of the literature regarding long-term outcomes of these infants and children.

Objective: To systematically review the literature on the long-term neurodevelopmental outcomes of infants and children born to mothers with acute parvovirus B19 infection in pregnancy.

Methods: Electronic databases MEDLINE, EMBASE, CINAHL, PsychInfo, Cochrane Database of Systematic Reviews (CDSR) and Cochrane Central Register of Controlled Trials (CCRT) were searched to identify studies that reported on long-term neurodevelopmental outcome of infants and children born following maternal parvovirus B19 infection in pregnancy. No language restrictions were applied. Data synthesis was performed using qualitative description and was categorized based on 1) outcome of infants and children with clinical presentation of non-immune hydrops fetalis and 2) outcomes of infants and children born to mothers with serologic evidence of acute infection.

Results: Eleven studies were included. Five studies reported on outcomes in infants and children with non-immune hydrops fetalis while six studies reported on outcomes in infants and children born to mothers with serologic evidence of acute infection. There is a suggestion that the incidence of adverse neurodevelopmental outcome is higher in those who present with non-immune hydrops fetalis [18\% (7/39), range from individual studies $0-31.3 \%$ ] compared to asymptomatic infants and children born to mothers with acute parvovirus B19 infection in pregnancy $[2.4 \%$ (12/494) range from individual studies $0-7.4 \%$ ].

Conclusion: Even though the outcome of parvovirus B19 in pregnancy in most cases is favorable, there is some supportive data that fetal parvovirus B19 infection can cause central nervous system damage. Future prospective studies needs to be conducted to address this issue.
\end{abstract}

\section{BACKGROUND}

Parvovirus B19, named from Latin "Parvum" meaning small, is a single-stranded DNA virus responsible for erythema infectiosum, also known as "slapped cheek disease" or "fifth disease" common in young children [1]. The virus was discovered in 1975 by Cossart et al. [2] during routine screening of serum for hepatitis B and identified as the cause of erythema infectiosum in 1983 [3]. Since then the link between maternal parvovirus B19 infection and nonimmune hydrops and fetal death has been well established [4-7].

The virus attaches to the "P" antigen that is linked to intracellular lytic and/or apoptotic pathways leading to cellular destruction and permitting viral replication [8]. The "P" antigen has been identified on erythroid progenitor cells, megarkaryocytes, endothelial cells, placental cells and fetal liver and myocardial cells which are involved in the complex of symptoms that characterize the infection. Parvovirus tend

*Address correspondence to this author at the Department of Paediatrics, Room 775A, Mount Sinai Hospital, 600 University Avenue, Toronto, Ontario M5G 1X8, Canada; Tel: 416-586-4816; Fax: 416-586-8745;

E-mail: vshah@mtsinai.on.ca to be species specific and animal strains are not associated with disease in the humans [1].

Seroprevalent studies have documented that approximately $35-50 \%$ of woman of reproductive age are susceptible to parvovirus B19 infection and at risk of vertically transmitting the infection to the fetus $[9,10]$. Approximately $1-3 \%$ of susceptible pregnant women without a known history of exposure to parvovirus B19 will seroconvert during pregnancy [10-13]. Up to $50 \%$ of susceptible pregnant women exposed to parvovirus B19 through household contacts will develop seropositivity while the rate of seroconversion is $20-30 \%$ when the exposure occurs in day care centres or school [11-13].

The risk of transmission to the fetus has been reported in the range of $17-33 \%$ [14-16] but infection remains asymptomatic in most cases [1]. However, a wide range of adverse outcomes have been reported in association with intrauterine parvovirus B19 infection including: spontaneous abortions (rate of $14.8 \%$ if infected prior to 20 weeks gestation and $2.3 \%$ if the infection occurs after 20 weeks gestation) [1719]; occurrence of non-immune hydrops fetalis (NIHF) [11,14-18]; congenital anomalies including central nervous system, craniofacial and eye anomalies $[1,20]$. The presenting features on ultrasound include generalized edema/ana- 
Table 1. Guide to Interpretation of Parvovirus Serology Values

\begin{tabular}{|c|c|c|c|}
\hline Parvovirus B19 Ig G Result & Parvovirus B19 Ig M Result & Interpretation & Clinical Implication \\
\hline \hline Negative & Negative & No antibody detected & Susceptible to parvovirus B19 infection \\
\hline Positive & Negative & Indicative of past exposure & Not susceptible to parvovirus B19 infection \\
\hline Positive & Positive & Indicative of infection & Infection most likely within the last 2 to 3 months \\
\hline Negative & Positive & Indicative of infection & $\begin{array}{c}\text { Evidence of acute/recent infection with B19. } \\
\text { Consider repeat testing in 2 to 4 weeks }\end{array}$ \\
\hline
\end{tabular}

sarca, ascites, pleural and pericardial effusion as well as placental edema. Other ultrasonographic manifestations of B19 infection include placentomegaly, polyhydramnios and Doppler findings consistent with anemia. Various mechanisms have been implicated in the occurrence of NIHF including severe fetal anemia leading to hypoxia and high output cardiac failure, development of fetal viral myocarditis leading to cardiac failure and impaired hepatic function $[8,21]$.

The diagnosis of maternal parvovirus infection is based on clinical symptoms and signs and evidence of infection on serological testing for parvovirus specific immunoglobulin (Ig) G and Ig M. Table 1 provides a guide to interpretation of parvovirus Ig $\mathrm{G}$ and Ig M serology results in the diagnosis of infection [22]. However, serology may not be useful if infection is suspected after onset of hydrops fetalis due to the lag period between immune response and occurrence of hydrops. Therefore, the method of choice for detection of intrauterine infection is polymerase chain reaction (PCR) amplification of viral DNA via amniocentesis or cordocentesis [23].

Prevention of exposure is ideal, but not feasible, in susceptible pregnant individuals as the period of contagion is prior to the onset of symptoms. Women with confirmed exposure should be evaluated in a tertiary care unit by a maternal-fetal medicine specialist. The fetus should be assessed weekly for 8 to 10 weeks for evidence of anemia using middle cerebral artery Dopplers and for hydrops. If hydrops develops at or near term, consideration should be made for delivery or intrauterine transfusion. If hydrops develops in late first or early second trimester, when directed fetal therapy is impractical, management should be expectant or termination of pregnancy could be considered. Management of the hydropic fetus is by cordocentesis to assess fetal hemoglobin and reticulocyte count and facilitate intrauterine transfusion. Currently there are no randomized controlled trials to evaluate the best management for fetal hydrops secondary to parvovirus B19 infection but intrauterine transfusion appears to be a reasonable option $[24,25]$.

To date, there have been practice guidelines published in Canada [24] and UK [25] regarding management of parvovirus B19 infection in pregnancy but none of these statements systematically review the literature regarding the long-term neurodevelopmental outcomes of infants and children. Further, the impact of clinical presentation on long-term neurodevelopment has not been addressed. The availability of this information is vital to physicians in order to provide appropriate counseling regarding management and therapeutic options to the pregnant couple.
We undertook this systematic review to provide a summary on the long-term neurodevelopmental outcomes of infants and children born to mothers with parvovirus B19 infection in pregnancy.

\section{MATERIAL AND METHODS}

\section{Search Strategies}

A comprehensive literature search using the OVID search platform was conducted in the following databases: MEDLINE (1950 - March 2009), EMBASE (1980 - March 2009), CINAHL (1982 - March 2009), PsychInfo (1967 March 2009), Cochrane Database of Systematic Reviews (CDSR) and Cochrane Central Register of Controlled Trials (CCRT) $\left(1^{\text {st }}\right.$ Quarter 2009). No language restrictions were applied. The search terms used to identify studies for inclusion in this review are described in Appendix. The articles were scanned initially based on titles and abstracts by two reviewers (AJ, VS). Articles that reported on the long-term neurodevelopmental outcomes following maternal parvovirus B19 infection in pregnancy were retrieved in full and assessed for eligibility by two reviewers (AJ, VS). The reference lists of eligible studies were checked to identify additional studies for inclusion in this review. A $\log$ of search strategy, articles scanned, articles retrieved and included and excluded are reported in the results section.

\section{Inclusion Criteria}

Studies that reported on long-term neurodevelopmental outcome of infants and children born following maternal parvovirus B19 infection in pregnancy were included irrespective of the type of study design. We included cohort studies (prospective or retrospective), case-control studies, case series and case reports if they described the neuro-developmental outcomes. Information from studies published as full/short report was included.

\section{Exclusion Criteria}

Information presented as abstracts, letters, commentaries and editorials were excluded.

\section{Primary Outcome}

The primary outcome was neurodevelopmental outcome of infants and children born following maternal parvovirus B19 infection in pregnancy assessed using a validated tool or based on information provided by their physicians or caregivers using a questionnaire. Examples of validated tools include: Snijders-Oomen non-verbal intelligence test [26], Kaufmann assessment battery for children [27], Griffiths Test for infant development [28], Denver Developmental Screening test [29] and Bayley Scales of Infant Development [30]. 


\section{Data Abstraction and Presentation}

Data from each eligible study were extracted individually by two reviewers (AJ, VS) and compared. The reviewers resolved any disagreements through discussion or, if required, consulted a third person (EK).

Data synthesis was performed using qualitative description from the included studies and was categorized based on the clinical presentation: 1) infants and children with clinical presentation of NIHF and 2) infants and children born to mothers with serologic evidence of acute infection during pregnancy.

\section{RESULTS}

The electronic searches yielded 1034 citations from all six databases. All references were stored in an EndNote ${ }^{\mathrm{TM}}$ library that identified 178 duplicates. The remaining 856 unique references were screened by two authors (AJ, VS) against the inclusion criteria and 12 articles [14, 16, 17, 31-39] were retrieved for additional review (Fig. 1). Eleven studies [14, 16, 17, 31-36, 38, 39] met the eligibility criteria and were included in the systematic review. One study was excluded as it was a letter to the editor [37]. Information on the baseline characteristics of included studies is presented in Table 2. The results from studies are described below based on 1) outcomes of infants and children with NIHF and 2) confirmed maternal infection during pregnancy (based on maternal serology).

\section{Outcomes Based on Clinical Presentation of NIHF}

Five studies reported on the neurodevelopmental outcome in infants and children who presented with NIHF secondary to congenital parvovirus B19 infection (Table 2) [32-34, 36, 39]. The type of study designs include retrospective case series $(\mathrm{N}=3)[32,33,36]$ and case reports $(\mathrm{N}=2)[34,39]$. Results from individual studies are presented below.

Cameron et al. [32] reported outcome of 16 pregnancies complicated by NIHF secondary to parvovirus infection. Three fetuses received intrauterine transfusion (IUT) of packed red blood cells of which only one survived. The only live born infant in this study was followed up regularly and was reported to have normal development at 2 years of age.

Dembinski et al. [33] reported neurodevelopmental outcomes of infants and children with NIHF who received IUT from a single tertiary care center in Germany. Of the 67 confirmed cases of congenital infection (based on PCR or specific Ig M positive), 37 cases developed NIHF and received IUT. In this group, intrauterine deaths occurred in five while the remaining 32 were born alive with one infant dying in the neonatal period. Amongst survivors, the authors were able to obtain neurodevelopmental outcome in $20(35 \%$ loss to follow up). The age of infant at the time of assessment ranged from 1.08-9.1 years (median, 4.3 years). A variety of validated assessment tools were used to calculate the developmental quotient (DQ) and intelligence quotient (IQ) depending on the age of the child. For children $\leq 2$ years $(\mathrm{N}=3)$, Griffiths Test for Infant Development was used to calculate the DQ scores [mean 100, standard deviation (SD) 11]. Intelligence quotient was calculated using either the Kaufmann Assessment Battery for Children $>8$ years of age $(\mathrm{N}=3)$ or Snijders-Oomen Non-Verbal Intelligence Test for children between 2-6 years of age
$(\mathrm{N}=14)$ with a mean IQ of 100 and SD of 15 . The mean DQ/IQ score of all children was 101 (range 84-116). Two children had IQ/DQ score of 84 (below one SD) while one child had an IQ/DQ score that exceeded one SD. Neurological examination was reported to be normal in all children. Using a multivariate analysis, the following variables were found not to influence the IQ/DQ scores: minimum fetal haemoglobin $(\mathrm{p}=0.64)$ or fetal haematocrit $(p=0.97)$, gestational age at first IUT $(p=0.1)$ or at delivery $(\mathrm{p}=0.4)$ and number of IUT $(\mathrm{p}=0.1)$.

Donders et al. [34] reported neurodevelopmental outcome on a hydropic fetus secondary to parvovirus infection delivered at 31 weeks gestation for fetal distress. Following aggressive neonatal management and repeated red blood cell transfusion the infant recovered. The infant continued to have persistent anemia necessitating red blood cell transfusion until the age of 4 months. The infant had a positive test for PCR at 4 months which became negative on follow-up. The child was reported to have a normal neurodevelopmental outcome when assessed at 22 months [method of assessment not reported (NR)].

Nagel et al. [36] reported adverse neurodevelopmental outcome in fetuses with NIHF treated with IUT. Of the 24 cases in which IUT was performed, information on neurodevelopmental outcome was available in all survivors $(\mathrm{N}=16)$. Thirteen children were assessed by the investigators in the out- patient clinic; one was assessed at home while for the remaining two children who were evaluated elsewhere information was retrieved. Age of children at the time of follow up was between $0.4-8$ years. For infants and children between $0.08-3.5$ years, The Bayley Scale of Infant Development $(\mathrm{N}=9)$ was used to calculate the mental developmental index (MDI) and psychomotor developmental index (PDI). The Snijders-Oomen Non Verbal Intelligence Test was used to calculate IQ for children between 2.5-7 years of age $(\mathrm{N}=7)$. The mean (SD) for these scores (MDI, PDI, IQ) is 100 (15). Eleven children (68\%) had scores within normal limits with normal neurological examination. Three children had mild delay (defined as scores of 70-84) while two children had severe delay (defined as scores $<70$ ). All these children were also noted to have neurological deficits on examination. These deficits include: marked hypotonia of lower limbs $(\mathrm{N}=1)$, delayed fine motor coordination $(\mathrm{N}=2)$, marked hypertonia and hyperreflexia of upper limbs $(\mathrm{N}=1)$ and generalized hypotonia, ataxia and strabismus $(\mathrm{N}=1)$. The child with ataxia was further evaluated by MRI scan which showed cerebellar vermis atrophy. Metabolic and laboratory evaluation in this child did not detect any other etiology for the cerebellar vermis atrophy. No correlation was demonstrated between neurodevelopmental outcome and pre transfusion fetal haemoglobin $(\mathrm{p}=0.83)$, platelet count $(\mathrm{p}=0.15)$ and intrauterine $\mathrm{pH}(\mathrm{p}=0.53)$ at the time of cordocentesis.

Sheikh et al. [39] reported two cases of NIHF secondary to parvovirus infection which resolved spontaneously and were reported to be normal at one year of age.

In summary, only two included studies reported adverse neurodevelopmental outcomes in infants and children who presented with NIHF. [33,36] In the study by Dembinski et al. [33], even though $2 / 20(10 \%)$ children had mild delay (IQ/DQ score < 85), the authors concluded that these 
Table 2. Long Term Outcomes Following Maternal Parvovirus B19 Infection in Pregnancy

\begin{tabular}{|c|c|c|c|c|c|}
\hline $\begin{array}{l}\text { Study, Year, } \\
\text { Country and } \\
\text { Design }\end{array}$ & $\begin{array}{l}\text { Number of Cases } \\
\text { and Study Period }\end{array}$ & $\begin{array}{c}\text { Fetal } \\
\text { Intervention and } \\
\text { Outcome }\end{array}$ & $\begin{array}{l}\text { Age at Follow-Up } \\
\text { and Method of } \\
\text { Assessment }\end{array}$ & Results & Conclusion and Limitations \\
\hline \multicolumn{6}{|c|}{ Studies Reporting Outcomes Based on Clinical Presentation of NIHF ${ }^{1}$} \\
\hline $\begin{array}{l}\text { Cameron et al. } \\
\text { 1997, UK } \\
\text { Retrospective case } \\
\text { series }\end{array}$ & $\begin{array}{l}\mathrm{N}=17 \text { cases } \\
\mathrm{NIHF}(\mathrm{N}=16) \\
1986-1996\end{array}$ & $\begin{array}{l}\text { IUD }(\mathrm{N}=16) \\
\text { IUT }(\mathrm{N}=3) \\
\text { Survival }(\mathrm{N}=1 / 3)\end{array}$ & $\begin{array}{l}2 \text { years } \\
\text { NR }\end{array}$ & $\begin{array}{l}\text { Normal development } \\
(\mathrm{N}=1)\end{array}$ & $\begin{array}{l}\text { NIHF associated with poor } \\
\text { outcome in terms of } \\
\text { mortality } \\
\text { Interpretation regarding long- } \\
\text { term outcome limited due to } \\
\text { single survivor }\end{array}$ \\
\hline $\begin{array}{l}\text { Dembinski et al. } \\
\text { 2002, Germany } \\
\text { Retrospective case } \\
\text { series }\end{array}$ & $\begin{array}{l}\mathrm{N}=67 \text { cases } \\
\text { NIHF treated with } \\
\text { IUT }(\mathrm{N}=37) \\
\text { Of those treated with } \\
\text { IUT, } \mathrm{N}=31 \text { survived } \\
\text { and follow-up data } \\
\text { available for } \mathrm{N}=20 \\
\text { 1989-1998 }\end{array}$ & $\begin{array}{l}\text { Spontaneous } \\
\text { resolution }(\mathrm{N}=30) \\
\text { IUD following } \\
\text { IUT }(\mathrm{N}=5) \\
\text { Live births } \\
(\mathrm{N}=32) \\
\text { Neonatal death } \\
(\mathrm{N}=1 / 32)\end{array}$ & $\begin{array}{l}13 \text { to } 110 \text { months } \\
\text { (median } 52 \text { months) } \\
\text { Assessment tools: } \\
\text { Snijders-Oomen non- } \\
\text { verbal intelligence } \\
\text { test }(\mathrm{N}=14 \text { ) } \\
\text { Kaufmann assessment } \\
\text { battery for } \\
\text { children (N=3) } \\
\text { Griffiths Test for } \\
\text { infant development } \\
(\mathrm{N}=3)\end{array}$ & $\begin{array}{l}\text { Mild delay }(\mathrm{N}=2) \\
\text { Neurodevelopmental } \\
\text { scores ranged within two } \\
\text { standard deviation of a } \\
\text { normal population and } \\
\text { exceeded one standard } \\
\text { deviation in three } \\
\text { children }\end{array}$ & $\begin{array}{l}\text { Children with successful IUT } \\
\text { have good } \\
\text { neurodevelopmental outcome } \\
\text { Limitations: } \\
\text { 1) Conclusion based on small } \\
\text { number of participants } \\
\text { 2) Different values of scores } \\
\text { reported in the abstract and } \\
\text { the results section. In } \\
\text { addition, unclear whether } \\
\text { scores are reported as mean } \\
\text { or media value } \\
\text { 3) Missing } \\
\text { neurodevelopmental outcome } \\
\text { on } 11 / 31(35 \%) \text { children }\end{array}$ \\
\hline $\begin{array}{l}\text { Donders et al. } \\
\text { 1994, Belgium }\end{array}$ & $\begin{array}{l}\text { NIHF }(\mathrm{N}=1) \\
\text { Follow-up data }(\mathrm{N}=1) \\
\text { Study period NR }\end{array}$ & NIHF at 30 weeks & $\begin{array}{l}\text { Follow-up until } 22 \\
\text { months } \\
\text { Method of neuro- } \\
\text { developmental } \\
\text { assessment NR }\end{array}$ & Developmentally normal & $\begin{array}{l}\text { No definitive conclusions } \\
\text { based on a single case report }\end{array}$ \\
\hline $\begin{array}{l}\text { Nagel et al. 2007, } \\
\text { Netherlands } \\
\text { Retrospective case } \\
\text { series }\end{array}$ & $\begin{array}{l}\text { NIHF with IUT for } \\
\text { anemia or } \\
\text { thrombocytopenia } \\
(\mathrm{N}=24) \\
\text { Follow-up data } \\
(\mathrm{N}=16) \\
1997-2005\end{array}$ & $\begin{array}{l}\text { IUD during the } \\
\text { procedure }(\mathrm{N}=1) \\
\text { IUD after the } \\
\text { procedure }(\mathrm{N}=6) \\
\text { Death at birth } \\
(\mathrm{N}=1)\end{array}$ & $\begin{array}{l}0.5 \text { to } 8 \text { years } \\
\text { Assessment tools: } \\
\text { Bayley Scales of } \\
\text { Infant Development } \\
\text { or } \\
\text { Snijders-Oomen test }\end{array}$ & $\begin{array}{l}\text { Mild developmental } \\
\text { delay }(\mathrm{N}=3) \\
\text { Severe developmental } \\
\text { delay }(\mathrm{N}=2)\end{array}$ & $\begin{array}{l}\text { Fetal parvovirus B19 } \\
\text { infection may result in } \\
\text { central nervous system } \\
\text { damage } \\
\text { Limitations: } \\
\text { 1) Small sample size with a } \\
\text { wide range of follow-up age }\end{array}$ \\
\hline $\begin{array}{l}\text { Sheikh et al. } \\
\text { 1992, USA } \\
\text { Case report }\end{array}$ & $\begin{array}{l}\text { NIHF }(\mathrm{N}=2) \\
\text { Follow-up data }(\mathrm{N}=2) \\
\text { Study period: } \mathrm{NR}\end{array}$ & $\begin{array}{l}\text { Spontaneous } \\
\text { resolution } \\
\text { Preterm birth at } \\
32 \text { weeks }(\mathrm{N}=1)\end{array}$ & $\begin{array}{l}\text { Follow-up at } 9 \text { and } 18 \\
\text { months for case } 1 \text { and } \\
2 \text { respectively } \\
\text { Method of assessment } \\
\text { NR }\end{array}$ & Developmentally normal & $\begin{array}{l}\text { No definite conclusions can } \\
\text { be made based on } 2 \text { cases }\end{array}$ \\
\hline \multicolumn{6}{|c|}{ Studies Reporting Outcomes Based on Serologic Evidence of Acute Maternal Infection ${ }^{2}$} \\
\hline $\begin{array}{l}\text { Bruu and Flugsrud } \\
\text { 1994, Norway } \\
\text { Retrospective case } \\
\text { series }\end{array}$ & $\begin{array}{l}\text { Acute maternal } \\
\text { infection in } \\
\text { pregnancy based on } \\
\text { serology }(\mathrm{N}=19) \\
\text { Follow up data } \\
(\mathrm{N}=16) \\
1984-1986\end{array}$ & $\begin{array}{l}\text { Miscarriages } \\
(\mathrm{N}=1)\end{array}$ & $\begin{array}{l}2 \text { years } \\
\text { Information from } \\
\text { local health authority }\end{array}$ & $\begin{array}{l}\text { Normal development } \\
(\mathrm{N}=15) \\
\text { Hyperactivity and } \\
\text { delayed language }(\mathrm{N}=1)\end{array}$ & $\begin{array}{l}\text { No evidence of increased } \\
\text { adverse neurodevelopmental } \\
\text { outcome } \\
\text { Limitations: } \\
\text { 1) None had evidence of } \\
\text { congenital infection } \\
\text { 2) Small sample size } \\
\text { 3) Method of assessment not } \\
\text { reported }\end{array}$ \\
\hline $\begin{array}{l}\text { Gratacós et al. } \\
\text { 1995, Spain } \\
\text { Prospective cohort } \\
\text { study }\end{array}$ & $\begin{array}{l}\text { Acute maternal } \\
\text { infection in } \\
\text { pregnancy based on } \\
\text { serology }(\mathrm{N}=60) \\
\text { Live born infants } \\
(\mathrm{N}=56) \\
\text { Follow-up data } \\
(\mathrm{N}=55) \\
\text { Study period: dates } \\
\text { NR (duration } 2 \text { years) }\end{array}$ & $\begin{array}{l}\text { Miscarriages } \\
(\mathrm{N}=5) \text { and } \\
\text { confirmed } \\
\text { secondary to } \mathrm{B} 19 \\
\text { infection }(\mathrm{N}=1) \\
\text { Serological } \\
\text { evidence of } \\
\text { congenital } \\
\text { neonatal infection } \\
\text { at } 1 \text { year of age } \\
(\mathrm{N}=12 / 48 \text { tested) }\end{array}$ & $\begin{array}{l}1 \text { year } \\
\text { Information on neuro- } \\
\text { developmental } \\
\text { outcome obtained } \\
\text { from paediatrician }\end{array}$ & $\begin{array}{l}\text { No severe neuro- } \\
\text { developmental outcome } \\
\text { reported in any infant }\end{array}$ & $\begin{array}{l}\text { Adverse fetal outcome rare in } \\
\text { infants born to mothers with } \\
\text { acute parvovirus B19 } \\
\text { infection in pregnancy } \\
\text { Limitations: } \\
\text { 1) No standardized method } \\
\text { of neurodevelopmental } \\
\text { assessment with short } \\
\text { duration of follow-up } \\
\text { 2) No report of whether there } \\
\text { was mild to moderate } \\
\text { adverse neuro-developmental } \\
\text { outcome }\end{array}$ \\
\hline
\end{tabular}




\begin{tabular}{|c|c|c|c|c|c|}
\hline $\begin{array}{c}\text { Study, Year, } \\
\text { Country and } \\
\text { Design }\end{array}$ & $\begin{array}{l}\text { Number of Cases } \\
\text { and Study Period }\end{array}$ & $\begin{array}{c}\text { Fetal } \\
\text { Intervention } \\
\text { and Outcome }\end{array}$ & $\begin{array}{l}\text { Age at Follow-Up and } \\
\text { Method of Assessment }\end{array}$ & Results & Conclusion and Limitations \\
\hline \multicolumn{6}{|c|}{ Studies Reporting Outcomes Based on Serologic Evidence of Acute Maternal Infection ${ }^{2}$} \\
\hline $\begin{array}{l}\text { Koch et al. } \\
\text { 1993, USA } \\
\text { Prospective } \\
\text { cohort study }\end{array}$ & $\begin{array}{l}\text { Acute maternal } \\
\text { infection in } \\
\text { pregnancy based on } \\
\text { serology }(\mathrm{N}=19) \\
\text { Live born infants } \\
(\mathrm{N}=19) \\
\text { Follow-up data } \\
(\mathrm{N}=19) \\
1990-1991\end{array}$ & $\begin{array}{l}\text { No evidence of } \\
\text { fetal hydrops or } \\
\text { death }\end{array}$ & $\begin{array}{l}\text { 3-21 months } \\
\text { Denver developmental } \\
\text { screening }\end{array}$ & Normal examination & $\begin{array}{l}\text { Acute parvovirus B19 } \\
\text { infection in pregnancy can be } \\
\text { associated with normal } \\
\text { neurodevelopmental outcome } \\
\text { Limitations: } \\
\text { 1) Information limited to } \\
\text { small sample size } \\
\text { 2) No information on scores } \\
\text { obtained form the screening } \\
\text { tool }\end{array}$ \\
\hline $\begin{array}{l}\text { PHL Service } \\
\text { Working Party on } \\
\text { Fifth Disease 1990, } \\
\text { UK } \\
\text { Prospective cohort } \\
\text { study }\end{array}$ & $\begin{array}{l}\text { Acute maternal } \\
\text { infection in } \\
\text { pregnancy based on } \\
\text { serology }(\mathrm{N}=190) \\
\text { Live births }(\mathrm{N}=156) \\
\text { Follow-up data } \\
\text { (N=114) } \\
1985-1988\end{array}$ & $\begin{array}{l}\text { Therapeutic } \\
\text { abortion }(\mathrm{N}=4) \\
\text { Spontaneous } \\
\text { abortion or } \\
\text { IUD }(\mathrm{N}=29) \\
\text { Stillbirth }(\mathrm{N}=1) \\
\text { Preterm }(\mathrm{N}=6)\end{array}$ & $\begin{array}{l}1 \text { year } \\
\text { Information on neuro- } \\
\text { developmental outcome } \\
\text { obtained from family } \\
\text { physicians using } \\
\text { questionnaire } \\
{ }^{3} \text { Further follow-up of } \\
129 / 156 \text { children } 7-10.5 \\
\text { years } \\
\text { Information on neuro- } \\
\text { developmental outcome } \\
\text { obtained from family } \\
\text { physicians using } \\
\text { questionnaire }\end{array}$ & $\begin{array}{l}\text { No developmental delay } \\
\text { in any infant } \\
\text { Developmental delay } \\
(\mathrm{N}=3)\end{array}$ & $\begin{array}{l}\text { Majority of the children will } \\
\text { have normal } \\
\text { neurodevelopment } \\
\text { Limitations: } \\
\text { 1) Information based on } \\
\text { questionnaire only rather } \\
\text { than formal assessment }\end{array}$ \\
\hline $\begin{array}{l}\text { Miller et al. } \\
\text { 1998, UK } \\
\text { Prospective cohort } \\
\text { study }\end{array}$ & $\begin{array}{l}\text { Acute maternal } \\
\text { infection in } \\
\text { pregnancy based on } \\
\text { serology }(\mathrm{N}=255) \\
\text { Live born infants } \\
(\mathrm{N}=215) \\
\text { Follow-up data } \\
(\mathrm{N}=182) \\
1992-1995\end{array}$ & $\begin{array}{l}\text { Therapeutic } \\
\text { abortion } \\
(\mathrm{N}=11) \\
\text { Spontaneous } \\
\text { abortion or } \\
\text { fetal death } \\
(\mathrm{N}=29)\end{array}$ & $\begin{array}{l}\text { At least } 1 \text { year of age } \\
\text { Information on neuro- } \\
\text { developmental outcome } \\
\text { obtained from family } \\
\text { physicians using } \\
\text { questionnaire }\end{array}$ & $\begin{array}{l}\text { Mild developmental } \\
\text { delay }(\mathrm{N}=3)\end{array}$ & $\begin{array}{l}\text { Majority of the children will } \\
\text { have normal } \\
\text { neurodevelopment } \\
\text { Limitations: } \\
\text { 1) Information based on } \\
\text { questionnaire only rather } \\
\text { than formal assessment and } \\
\text { short follow-up duration }\end{array}$ \\
\hline $\begin{array}{l}\text { Rodis et al. } 1998, \\
\text { USA } \\
\text { Retrospective case- } \\
\text { control study }\end{array}$ & $\begin{array}{l}\text { Cases: Women with } \\
\text { serologic evidence } \\
\text { of recent parvovirus } \\
\text { infection }(\mathrm{N}=113) \\
\text { Follow-up data } \\
(\mathrm{N}=108) \\
\text { Control: Pregnant } \\
\text { women who were } \\
\text { non-immune or } \\
\text { with serologic } \\
\text { evidence of past } \\
\text { infection ( } \mathrm{N}=99) \\
\text { Follow-up data } \\
(\mathrm{N}=97) \\
\text { 1988-1994 }\end{array}$ & $\begin{array}{l}\text { Cases: } \\
\text { Spontaneous } \\
\text { abortion }(\mathrm{N}=4) \\
\text { Stillbirth }(\mathrm{N}=1) \\
\text { Ectopic } \\
\text { pregnancy } \\
(\mathrm{N}=1) \\
\text { Twins }(\mathrm{N}=2) \\
\text { NIHF }(\mathrm{N}=2) \\
\text { Control: } \\
\text { Spontaneous } \\
\text { abortion }(\mathrm{N}=5) \\
\text { Stillbirth }(\mathrm{N}=2) \\
\text { Twins }(\mathrm{N}=5)\end{array}$ & $\begin{array}{l}6 \text { months to } 7 \text { years } \\
\text { Information based on } \\
\text { questionnaire to } \\
\text { caregivers (mothers) }\end{array}$ & $\begin{array}{l}\text { Cases: } \\
\text { Delays in motor, speech } \\
\text { or language development } \\
\text { or significant attention } \\
\text { deficits require special } \\
\text { education }(\mathrm{N}=8) \\
\text { Cerebral palsy }(\mathrm{N}=2) \\
\text { Motor delays }(\mathrm{N}=1) \\
\text { Attention, speech, } \\
\text { learning delay ( } \mathrm{N}=5) \\
\text { Both language and motor } \\
\text { delay }(\mathrm{N}=2) \\
\text { Controls: } \\
\text { Significant } \\
\text { developmental delays } \\
\text { (N=7) } \\
\text { Cerebral palsy }(\mathrm{N}=0) \\
\text { Motor delays }(\mathrm{N}=3) \\
\text { Attention, speech, } \\
\text { learning delay }(\mathrm{N}=3) \\
\text { Both language and motor } \\
\text { delay ( } \mathrm{N}=1) \\
\text { No statistically } \\
\text { significant difference in } \\
\text { the proportion of } \\
\text { developmental delay } \\
\text { between cases and } \\
\text { control ( }>0.05 \text { ) }\end{array}$ & $\begin{array}{l}\text { No apparent increase in the } \\
\text { occurrence of adverse } \\
\text { neurodevelopmental } \\
\text { outcomes in children exposed } \\
\text { to in utero parvovirus B19 } \\
\text { infection } \\
\text { Limitations: } \\
\text { 1) Inadequate power to } \\
\text { demonstrate a two fold } \\
\text { difference in the risk of } \\
\text { adverse neurodevelopmental } \\
\text { outcome between groups }\end{array}$ \\
\hline
\end{tabular}

IUD: Intrauterine death; IUT: Intrauterine transfusion; NIHF: Non-immune hydrops fetalis; NR: Not reported.

${ }^{1}[32-34,36,39]$.

${ }^{2}[14,16,17,31,35,38]$.

${ }^{1}$ Further follow-up data at 7-10.5 years obtained using same methodology and reported in a separate publication by Miller et al. [17]. 

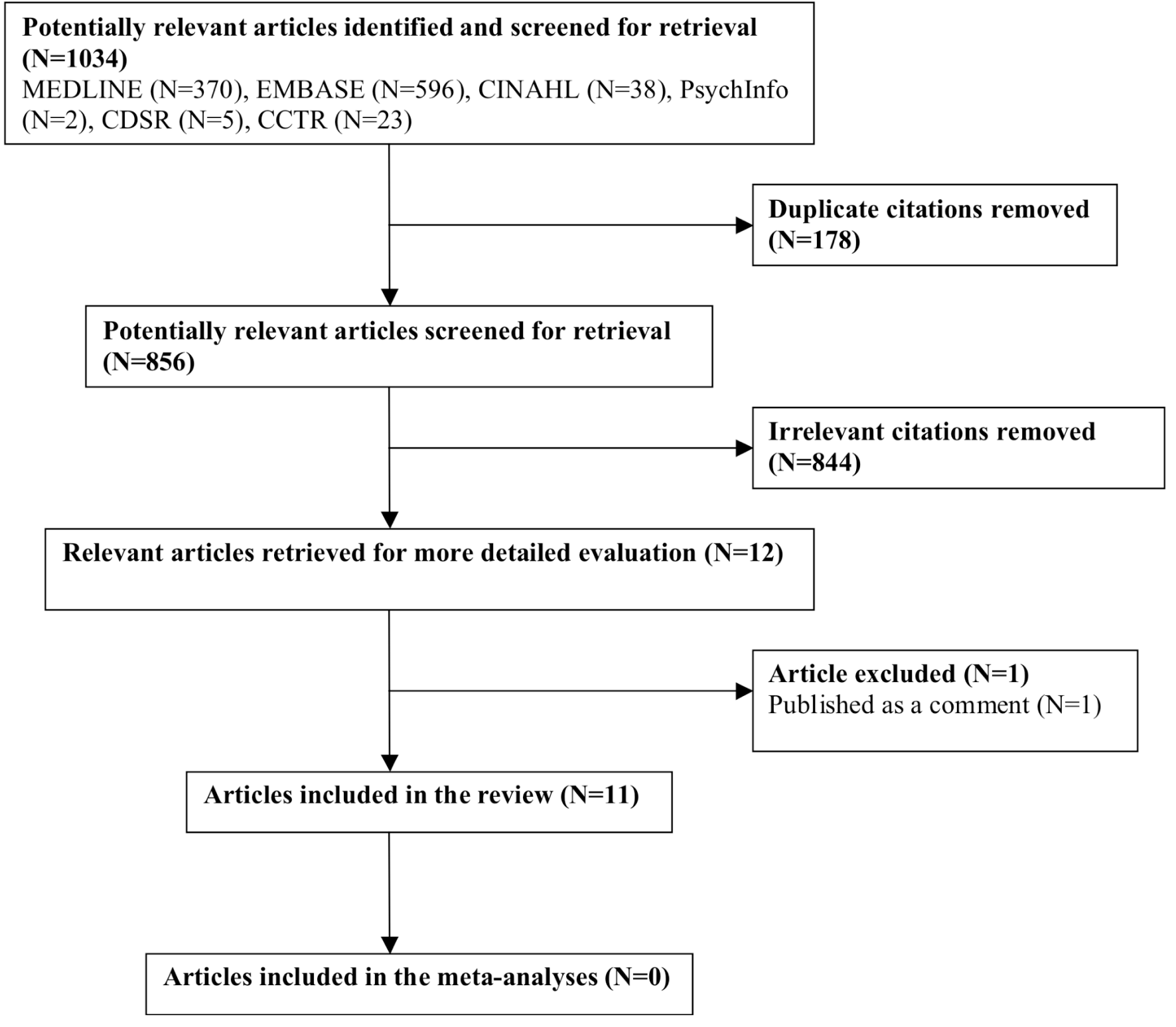

Fig. (1). Flow diagram for selection of studies.

findings were insignificant with all children having normal neurological examination. In contrast, Nagel et al. [36] reported that 5/16 (31\%) infants and children had evidence of delayed psychomotor development [3/16 (19\%) with mild delay and 2/16 (12\%) with severe delay]. All five of these infants and children had tone abnormalities with ataxia andstrabismus reported in one. None of these studies had a healthy control group for comparison. In total, 7/39 (18\%) infants and children who presented with NIHF had adverse neurodevelopmental outcome with a range of $0-31.3 \%$.

\section{Outcomes Based on Serologic Evidence of Acute Maternal Infection}

Six studies reported on the neurodevelopmental outcome in infants and children born to mothers with serologic evidence of acute parvovirus infection during pregnancy (Table 2) [14, 16, 17, 31, 35, 38]. The type of study designs include prospective cohort studies $(\mathrm{N}=4)[14,16,17,35]$, retrospective case series $(\mathrm{N}=1)$ [31], and case control study
$(\mathrm{N}=1)$ [38]. Results from individual studies are presented below.

Bruu and Flugsrud [31] reported on 19 pregnant women with serologic evidence of acute parvovirus infection. There were two cases of miscarriage. Follow up data was available for 16 children at two years of age. Except for one child with hyperactivity disorder and delayed language, all were reported to have normal growth and development. There was no evidence of congenital infection in these children based on serological testing.

Gratacós et al. [16] identified pregnant women with serologic evidence of acute parvovirus infection $(\mathrm{N}=60)$. The incidence of fetal loss secondary to parvovirus infection was reported to be $1.66 \%$. Of the five cases with spontaneous abortion, only one fetus was positive for PCR DNA. Six of the 56 live born infants (one was a twin pregnancy) were positive for Ig M antibodies suggesting congenital infection. Information was obtained for 55 of these infants at 1 year of age from paediatricians. None of the infants were reported to 
have severe neurodevelopmental impairment or congenital anomalies.

Koch et al. [35] prospectively evaluated 19 pregnant women with confirmed acute parvovirus infection (Ig M positive). All women delivered healthy term infants. Neonatal information was obtained retrospectively from medical records. Of the four infants who had serology testing at birth, three had evidence of congenital infection (Ig M positive). Follow-up evaluation included: physical and neurological examination, developmental assessment using Denver developmental screening and hematological testing. The age range of follow-up was $0.25-1.75$ years. All infants were reported to be normal with no evidence of anemia.

The first report on the long-term neurodevelopmental outcomes of infants born to pregnant women with acute parvovirus infection in pregnancy was published from UK. The study was a prospective cohort study conducted during 1985-1988 by Public health Laboratory (PHL) service working party on fifth disease [14]. Of the 190 pregnant women enrolled in the study, four underwent elective termination of pregnancy, 29 had either spontaneous abortion or intrauterine fetal death, and one pregnancy ended in stillbirth. Out of the 34, 14 fetuses were tested for B19 DNA and six were confirmed positive. Rate of fetal loss was more common if infection occurred before compared to after 20 weeks of gestation though the difference was not statistically significant $[17 \%$ vs $6 \%, \mathrm{p}=0.3$, relative risk (RR) $2.87,95 \%$ confidence interval (CI) 0.42 to 19.78$]$. Out of 129 infants eligible for follow up (age $\geq$ one year), information was obtained from family physicians in 114 cases $(12 \%$ loss to follow up). Details on infants' physical and neurodevelopment was obtained by requesting family physicians to complete a simple form. No details were provided regarding the method of assessment. None of the infants had any congenital malformation or serious neurodevelopmental problem reported. Of the 114 infants, 21 (18\%) had some evidence of congenital parvovirus infection (Ig G positive at 1 year).

In a subsequent study using the same methodology and questionnaire, Miller et al. [17] conducted a prospective cohort study following an outbreak of erythema infectiosum in UK between 1992 and 1995. Information was obtained regarding neurodevelopmental outcome from 182 infants at one year of age. The rate of mild delay was $1.6 \%(\mathrm{~N}=3)$. Evidence of congenital infection was found in $20 \%$ of infants tested (salivary sample positive of $\mathrm{Ig} G$ at 1 year of age).

Miller et al. [17] also obtained neurodevelopmental outcome from family physicians of 129 children who were enrolled in the PHL study published in 1990 [14]. The rate of some developmental delay was $2 \%(\mathrm{~N}=3)$ at the age of 7 10.5 years.

In a retrospective case-control study, Rodis et al. [38] compared neurodevelopmental outcomes of children born to 113 pregnant women with evidence of recent parvovirus infection during pregnancy (Ig M positive) to 99 pregnant women with evidence of past infection ( $\mathrm{Ig} G$ positive) or who were susceptible to infection (both Ig $\mathrm{G}$ and Ig M negative) during the same period. There were two cases of NIHF in study group (one was a still birth while the second survived following IUT) compared to none in control group. The median age (range) of follow-up was $4(0.5-7)$ years. Neurodevelopmental outcomes were available in 108 children (cases) compared to 97 (controls) from mothers using a questionnaire. There was no statistically significant difference in the rate of children with developmental delays between the study and control group $(7.4 \%$ vs $7.2 \%, \mathrm{p}>0.05)$. Two infants in the study group developed cerebral palsy (one with mild spasticity and other with severe ataxic cerebral palsy) compared to none in the control group. Difference in occurrence of cerebral palsy was not statistically significant.

In summary, 3 of the 6 included studies reported adverse neurodevelopmental outcomes in infants and children born to mothers with serologic evidence of acute infection. Bruu and Flugsrud [31] reported hyperactivity and delayed language in $1 / 16(6.2 \%)$ children. Miller et al. [17] reported $3 / 182(1.6 \%)$ children with mild delay. Rodis et al. [38] reported adverse outcome in 8/108 (7.4\%) children, however when compared to a control group the incidence was not statistically different. Two of the study infants had cerebral palsy compared to none in the control group which the authors found concerning. Miller et al. [17] also obtained information at 7-10 years of age for the original cohort of PHL study [14] and reported the incidence of some delay as $3 / 129$ (2\%) children (this cohort was reported by PHL to be normal at 1 year of age). The only study [35] that evaluated neurodevelopmental outcome using validated tools did not report any abnormalities in the study population. The other 2 included studies $[14,16]$ only reported on absence or presence of severe adverse neurodevelopmental outcomes. In total, $12 / 494(2.4 \%)$ infants and children who were born to mothers with serologic evidence of acute infection had adverse neurodevelpmental outcome with a range of $0-7.4 \%$.

\section{DISCUSSION}

In this review of combined data from studies selected on the basis of well defined inclusion criteria, there is a suggestion that the incidence of adverse neurodevelopmental outcome is higher in infants and children who present with NIHF compared to infants born to mothers with serologic evidence of acute parvovirus infection. Of those with adverse neurodevelopmental outcome, delayed psychomotor development and tone abnormalities seemed to predominate. Methodological challenges of this review include data derived from few studies with small number of participants, not all infants with NIHF received IUT, the diagnostic criteria of congenital parvovirus infection in the neonate was heterogeneous, there was a marked variation in the methods used to assess neurodevelopmental outcome (ranging from validated assessment tools to information derived from parents and physicians using questionnaire or simply reporting that these infants and children were followed at regular intervals), variation in reporting adverse outcomes (i.e. some studies reported on the presence or absence of severe outcome), wide range of age at which neurodevelopmental outcome was assessed making comparison between studies difficult and in most studies a healthy control group was not included as a comparator. In addition, neurodevelopmental outcome was not available for all infants and children with NIHF or those born to mothers with acute parvovirus B19 infection from the initial cohort. Therefore, caution should be exercised in interpreting this finding as the conclusion is based on included studies with methodological differences. 
Of the five studies [32-34, 36, 39] that report on outcomes in infants with NIHF, adverse neurodevelopmental outcome was reported in two studies [33-36]. Five of the 16 (31.3\%) survivors had adverse outcome which included mild and severe delay in three and two children respectively in the study by Nagel et al. [36] In addition, all these children had neurological deficits. In the study by Dembinski et al. [33] 2 children were reported to have mild delay $2 / 20(10 \%)$ however in this case series $35 \%$ of subjects were lost to follow-up. Parents of infants and children with adverse outcome are less likely to be compliant with their follow-up visits and this could have influenced the results of this study [40]. Further, in both these studies there was a wide range in age of follow up and different assessment tools were used. In the remaining three studies [32, 34, 39], the conclusion of normal development was based on case reports/series. Based on individual study information, the range of adverse neurodevelopmental outcome was $0 \%$ to $31.3 \%$.

Six studies report on neurodevelopmental outcome in infants and children born to mothers with serologic evidence of acute parvovirus B19 infection in pregnancy. The type of outcomes reported varied from no severe adverse outcome $[14,16]$ to delays in motor, speech or language development or significant attention deficits requiring special education [17, 31, 38]. Except for the study by Koch et al. [35] in which Denver developmental testing was used to assess outcomes, in all the remaining studies information was obtained using questionnaire either from physicians, local health authority or caregivers. None of the studies described the type of questionnaire used. This could put the validity of the study in question. The range of adverse neurodevelopmental outcome varies from $0 \%$ to $7.4 \%$ based on individual studies.

The mechanisms by which parvovirus B19 can cause central nervous system damage is unclear. Postulated mechanisms include teratogenic effect [41, 42], vasculitis [43], hypoxicischemic effects secondary to anemia and intracranial bleeding secondary to thrombocytopenia [44]. In animals, parvovirus B19 has a teratogenic effect causing cerebellar hypoplasia and ataxia in cats and anencephaly, microcephaly, facial defects and ectopic hearts in hamsters [41, 42]. Several studies have reported on the neuropathological findings in fetuses, neonates and adults with parvovirus B19 infection. The findings include development of fetal ventriculomegaly [45] and presence of multinucleated giant cells predominantly in the cerebral white matter and calcifications with a perivascular predilection in the white matter, cerebral cortex, basal ganglia, thalamus and germinal layer. Parvovirus B19 genome DNA was detected in the nucleus of the multinucleated giant cells by PCR amplification and in situ PCR methods [46]. Barah et al. [47] tested cerebrospinal fluid samples from 162 patients (1 day to 15 years of age) with undiagnosed meningoencephalitis who presented during an outbreak of parvovirus B19. Seven patients were found to be positive for parvovirus (4.3\%) [47]. In a subsequent publication from the same population, Kerr et al. documented elevated cytokine levels in serum and cerebrospinal fluid in 12 subjects with proven B19 meningoencephalitis [48]. Two of these subjects were newborns with possible perinatally acquired infection. In four cases cerebellar involvement was suggested either clinically (ataxia) or pathologically (atrophy of the molecular and granular layers of the cerebellum with focal loss of Purkinjie cells). Neuroimaging (MRI or CT) was performed in six cases and showed evidence of demyelination. The consequences of anemia secondary to parvovirus B19 can be comparable to neonates with blood group incompatibility where adverse neurodevelopmental outcome have been reported to be in the range of $4.5 \%$ to $10 \%$ [49-51]. Therefore, one may speculate that the a combination of each of the above pathophysiological mechanisms may be implicated in the occurrence of adverse neurodevelopmental outcome.

Despite a comprehensive review of the literature, we conclude that there is paucity of systematically collected data regarding long-term outcome of children following parvovirus B19 infection on which to base evidence based counseling. However, in spite of these concerns it can be concluded that the outcome of parvovirus B19 in pregnancy is favorable in most cases. Most women exposed to parvovirus B19 do not become infected and most infected mothers will not transmit the infection to the fetus. Further, most infected fetuses remain asymptomatic and do not become hydropic. With the availability of IUT, survival of hydropic fetus has improved. There is some data suggesting that fetal parvovirus B19 infection can cause central nervous system damage.

In order to obtain definitive evidence regarding long-term neurodevelopmental outcome with parvovirus B19 infection, future studies with adequate sample size using standardized neurodevelopmental assessment tools at 18-24 months and school age need to be conducted. In addition, the role of neuroimaging such as MRI (both fetal and neonatal) in identifying the effects of parvovirus B19 on cranial structures should be elucidated. These findings should be correlated with neurodevelopmental outcomes to provide appropriate counseling to women with acute parvovirus infection in pregnancy. It would be unethical to conduct a randomized trial evaluating the efficacy of IUT in fetuses with anemia as its role in improving mortality is already proven. Due to the rarity of number of cases born to mothers with acute parvovirus B19 infection seen at each center/year, a multicenter prospective cohort trial will be needed to address this issue.

\section{DEFINITIONS}

Susceptible Individual: An individual without evidence of previous immunity against parvovirus B 19 (i.e. absence of $\mathrm{Ig} \mathrm{G}$ ) and therefore at risk of developing infection if exposed to the virus.

History of Exposure: A significant contact with a person suffering from acute parvovirus B19 infection. Significant contact is defined as contact in the same room (e.g. in a house or classroom or a 2-4 bed hospital stay) for a significant period of time (15 minutes or more), or face-toface contact with a laboratory confirmed case of parvovirus B19 infection during the period of maximum infectivity (from 7 days prior to appearance of a rash to the date of appearance of the rash) in the absence of respiratory isolation precautions [52].

\section{ACKNOWLEDGEMENT}

We would like to acknowledge Ms. Elizabeth Uleryk, chief librarian at The Hospital for Sick Children who performed the literature search for this review. 


\section{APPENDIX: LITERATURE SEARCH STRATEGY}

We ran searches using the OVID search platform in the following databases: MEDLINE, EMBASE, CINAHL, PsycINFO, CDSR and CCTR. We retrieved a total of $\mathbf{1 0 3 4}$ references from all 6 databases. All references were saved in an EndNote library used to identify the $\mathbf{1 7 8}$ duplicates. Author AJ and VS reviewed the remaining $\mathbf{8 5 6}$ unique references against our inclusion criteria.

The following tables and text record the search strategies and terms used.

\section{MEDLINE}

The search strategy for MEDLINE (1950 to March Week 3 2009) retrieved 370 references of which 370 were unique and not duplicated in our other searches. We used a combination of MeSH and free text terms for

\begin{tabular}{|c|c|c|c|}
\hline Set \# & History & Results & Comments \\
\hline 1 & parvoviridae/ or parvovirus b19, human/ or Erythema Infectiosum/ & 3619 & Parvovirus MeSH terms \\
\hline 2 & exp pregnancy complications/ or exp Pregnancy/ & 633863 & Pregnancy MeSH terms \\
\hline 3 & exp "congenital, hereditary, and neonatal diseases and abnormalities"/ & 792727 & Congenital Abnormality MeSH terms \\
\hline 4 & 1 and 2 and 3 & 305 & Congenital abnormalities base set \\
\hline 5 & $\begin{array}{l}\text { exp mental disorders/ or exp "behavior and behavior mechanisms"/ or exp } \\
\text { "psychological phenomena and processes"/ or exp neurologic manifestations/ or exp } \\
\text { human development/ or exp fetal alcohol syndrome/ or exp Nervous System/ or exp } \\
\text { Nervous System Diseases/ or child development/ or exp diagnostic techniques, } \\
\text { neurological/ or exp neuropsychological tests/ or cerebral arteries/ or } \\
\text { cerebrovascular circulation/ or brain chemistry/ or exp nerve growth factors/ or exp } \\
\text { nervous system physiology/ or exp neurotransmitter agents/ or exp receptors, } \\
\text { neurotransmitter/ or circadian rhythm/ or mental health/ or exp psychological tests/ } \\
\text { or exp social behavior disorders/ or child psychiatry/ or exp psychophysiology/ or } \\
\text { exp neuropsychological tests/ or hallucinations/ or illusions/ or fetal alcohol } \\
\text { syndrome/ or exp psychology/ or communication/ or language/ or exp verbal } \\
\text { behavior/ or exp nonverbal communication/ or exp child development/ or fetal } \\
\text { alcohol syndrome/ or exp movement/ or exp nervous system physiology/ or exp } \\
\text { vision tests/ }\end{array}$ & 5016213 & Neurocognitive MeSH terms \\
\hline 6 & 1 and 5 & 221 & Neurocognitive outcomes base set \\
\hline 7 & 4 or 6 & 370 & Total unique citations \\
\hline
\end{tabular}

\section{EMBASE}

The search strategy for EMBASE (1980 to 2009 Week 12) retrieved 596 references of which 473 were unique and not duplicated in our other searches. There were $\mathbf{1 2 3}$ duplicates from MEDLINE. We used a combination of EMBASE and free text terms for

The following EMBASE search strategy retrieved 596 citations of which 123 were duplicates from MEDLINE

\begin{tabular}{|c|l|c|c|}
\hline Set \# & \multicolumn{1}{|c|}{ History } & Results & Comments \\
\hline \hline 1 & parvovirus/ or Erythema Infectiosum/ or "parvovirus b19".mp. (4720) & 4720 & Parvovirus EMBASE terms \\
\hline 2 & exp pregnancy/ or exp fetus disease/ or exp pregnancy complication/ & 220475 & Pregnancy EMBASE terms \\
\hline 3 & $\begin{array}{l}\text { exp Congenital Disorder/ or exp Prenatal Disorder/ or prenatal exposure/ or } \\
\text { exp postnatal development/ or }\end{array}$ & 402039 & Congenital Abnormality EMBASE terms \\
\hline 4 & \multicolumn{1}{|c|}{ 1 and 2 and 3 } & 414 & Congenital abnormalities base set \\
\hline 5 & $\begin{array}{l}\text { exp "Disorders of Higher Cerebral Function"/ or exp motor dysfunction/ or } \\
\text { exp sensory dysfunction/or exp speech disorder/ or exp mental function/or } \\
\text { Cerebral Palsy/ or exp communication disorder/ or exp psychomotor disorder/ } \\
\text { or sensation/ or neurocogniti*ti,ab. }\end{array}$ & 1476624 & Neurocognitive EMBASE terms \\
\hline 6 & 1 and 2 and 5 & 19 & Neurocognitive outcomes base set \\
\hline 7 & 4 or 6 & 596 & Total unique citations \\
\hline
\end{tabular}




\section{CINAHL (EBSCOhost)}

The search strategy for CINAHL (1982 to March Week 3 2009) retrieved 38 references of which 7 were unique and not duplicated in our other searches. We used a combination of CINAHL and free text terms for

\begin{tabular}{|c|l|c|c|}
\hline Set \# & \multicolumn{1}{|c|}{ History } & Results & Comments \\
\hline \hline 1 & (MH "Parvovirus Infections+") & 2120 & Parvovirus CINAHL terms \\
\hline 2 & (MH "Pregnancy+") or (MH "Pregnancy Complications+") & 64853 & Pregnancy CINAHL terms \\
\hline 3 & (MH "Neonatal, Fetal Diseases and Abnormalities (Non-Cinahl)+") & 39982 & Congenital Abnormality CINAHL terms \\
\hline 4 & 1 and 2 and 3 & 33 & Congenital abnormalities base set \\
\hline 5 & $\begin{array}{l}\text { (MH "Delirium, Dementia, Amnestic, Cognitive Disorders+") or (MH } \\
\text { "Behavior and Behavior Mechanisms+") or (MH "Behavioral and Mental } \\
\text { Disorders (Non-Cinahl)+") or (MH "Neurodegenerative Diseases+") or (MH }\end{array}$ & 694960 & Neurocognitive CINAHL terms \\
\hline 6 & "Nervous System Diseases+") & 7 & Neurocognitive outcomes base set \\
\hline 7 & 1 and 2 and 5 & 38 & Total unique citations \\
\hline
\end{tabular}

\section{PsycINFO}

The search strategy for PsycINFO (1967 to March Week 3 2009) retrieved 2 citations of which 1 was a duplicate from MEDLINE, EMBASE and CINAHL

\begin{tabular}{|c|c|c|c|}
\hline Set \# & History & Results & Comments \\
\hline \hline 1 & parvovir*.mp. & 2 & Parvovirus textword terms \\
\hline
\end{tabular}

\section{EBM Reviews - Cochrane Database of Systematic Reviews}

The following CDSR (1st Quarter 2009) search strategy retrieved 5 citations none of which were duplicated in MEDLINE, EMBASE, CINAHL and PsycINFO

\begin{tabular}{|c|c|c|c|}
\hline Set \# & History & Results & Comments \\
\hline \hline 1 & parvovir*.mp. & 5 & Parvovirus textword terms \\
\hline
\end{tabular}

\section{EBM Reviews - Cochrane Central Register of Controlled Trials}

The following CCTR (1st Quarter 2009) search strategy retrieved 23 citations all of which were duplicated in MEDLINE, EMBASE, CINAHL, and PsycINFO, but not CDSR

\begin{tabular}{|c|c|c|c|}
\hline Set \# & History & Results & Comments \\
\hline \hline 1 & parvovir*.mp. & 23 & Parvovirus textword terms \\
\hline
\end{tabular}

\section{REFERENCES}

[1] Levy R, Weissman A, Blomberg G, Hagay ZJ. Infection by parvovirus B 19 during pregnancy: a review. Obstet Gynecol Surv 1997; 52: 254-9.

[2] Cossart YE, Field AM, Cant B, Widdows D. Parvovirus-like particles in human sera. Lancet 1975; 1: 72-3.

[3] Anderson MJ, Jones SE, Fisher-Hoch SP, et al. Human parvovirus, the cause of erythema infectiosum (fifth disease)? Lancet 1983; 1: 1378.

[4] Brown T, Anand A, Ritchie LD, Clewley JP, Reid TM. Intrauterine parvovirus infection associated with hydrops fetalis. Lancet 1984; 2: 1033-4.

[5] Knott PD, Welpy GA, Anderson MJ. Serologically proven intrauterine infection with parvovirus. BMJ 1984; 289: 1660.
[6] Kinney JS, Anderson LJ, Farrar J, et al. Risk of adverse outcomes of pregnancy after human parvovirus B 19 infection. J Infect Dis 1988; 157: 663-7.

[7] Rodis JF, Hovick TJ, Quinn DL, Rosengren SS, Tattersall P. Human parvovirus infection in pregnancy. Obstet Gynecol 1988; 72: 733-8.

[8] Young NS, Brown KE. Parvovirus B19. N Engl J Med 2004; 350 586-97.

[9] Cohen BJ, Buckley MM. The prevalence of antibody to human parvovirus B19 in England and Wales. J Med Microbiol 1988; 25: 151-3.

[10] Valeur-Jensen AK, Pedersen CB, Westergaard T, et al. Risk factors for parvovirus B19 infection in pregnancy. JAMA 1999; 281: 1099-105.

[11] Leads from the MMWR. Risks associated with human parvovirus B19 infection. JAMA 1989; 261: 1406-8. 
[12] Gillespie SM, Cartter ML, Asch S, et al. Occupational risk of human parvovirus B19 infection for school and day-care personnel during an outbreak of erythema infectiosum. JAMA 1990; 263: 2061-5.

[13] Chorba T, Coccia P, Holman RC, et al. The role of parvovirus B 19 in aplastic crisis and erythema infectiosum (fifth disease). J Infect Dis 1986; 154: 383-93.

[14] Public Health Laboratory Service Working Party on Fifth Disease. Prospective study of human parvovirus infection in pregnancy. BMJ 1990; 300: 1166-70.

[15] Harger JH, Alder SP, Koch WC, Harger GF. Prospective evaluation of 618 pregnant women exposed to parvovirus B 19: risks and symptoms. Obstet Gynecol 1998 ;91: 413-20.

[16] Gratacós E, Torres PJ, Vidal J, et al. The incidence of human parvovirus B19 infection during pregnancy and its impact on perinatal outcome. J Infect Dis 1995; 171: 1360-3.

[17] Miller E, Fairley CK, Cohen BJ, Seng C. Immediate and long term outcome of human parvovirus B19 infection in pregnancy. $\mathrm{Br} \mathrm{J}$ Obstet Gynaecol 1998; 105: 174-8.

[18] Rodis JF, Quinn DL, Gary GW, et al. Management and outcomes of pregnancies complicated by human parvovirus B19 infection: a prospective study. Am J Obstet Gynecol 1990; 163: 1168-71.

[19] Cohen B. Parvovirus B19: an expanding spectrum of disease. BMJ 1995;311:1549-52.

[20] Markenson GR, Yancey MK. Parvovirus B19 infections in pregnancy. Semin Perinatol 1998; 22: 309-17.

[21] de Jong EP, de Haan TR, Kroes AC, Beersma MF, Opekes D, Walther FJ. Parvovirus B19 infection in pregnancy. J Clin Virol 2006; 36: 1-7.

[22] McCarter-Spaulding D. Parvovirus B 19 in pregnancy. J Obstet Gynecol Neonatal Nurs 2002; 31: 107-11.

[23] Riley LE, Fernandes CJ. Parvovirus B 19 infection during pregnancy. UpToDate 2008: Availaible from: http://www.utdol.com/ online/content/topic.do?topicKey=viral in/12853\&selectedTitle $=1$ $\sim 150 \&$ source $=$ search_result. [Accessed on: 16 June 2009].

[24] Crane J. Society of Obstetricians and Gynaecologists of Canada. Parvovirus B19 infection in pregnancy. J Obstet Gynaecol Can 2002; 24: 727-43.

[25] Morgan-Capner P, Crowcroft NS. PHLS Joint Working Party of the Advisory Committees of Virology and Vaccines and Immunization. Guidelines on the management of,and exposure to,rash illness in pregnancy (including consideration of relevant antibody screening programmes in pregnancy. Commun Dis Public Health 2002; 5: 59-71.

[26] Harris SH. An evaluation of the Snijders-Oomen nonverbal intelligence scale for young children. J Pediatr Psychol 1982; 7: 239-51.

[27] Kaufman AS, O'Neal MR, Avant AH, Long SW. Introduction to the Kaufman Assessment Battery for Children (K-ABC) for pediatric neuroclinicians. J Child Neurol 1987; 2: 3-16.

[28] Griffiths R. The abilities of young children: a comprehensive system of mental measurement for the first 8 years of life. The Test Agency, High Wycombe, UK 1970.

[29] Frankenburg WK, Dobbs JB. The denver developmental screening test. J Pediatr 1967; 71: 181-91.

[30] Bayley N. Bayley scales of infant development. 2 ${ }^{\text {nd }}$ Ed. San Antonio, TX: Psychological Corp. 1993.

[31] Bruu AL, Flugsrud LB. Erythema infectiosum in pregnancy: a follow-up of children after 2 years. Tidsskr Nor Laegeforen 1994; 114: 308-10

[32] Cameron AD, Swain S, Patrick WJ. Human parvovirus B19 infection associated with hydrops fetalis. Aust N Z J Obstet Gynaecol 1997; 37: 316-9.

[33] Dembinski J, Haverkamp F, Maara H, Hansmann M, Eis-Hübinger AM, Bartmann P. Neurodevelopmental outcome after intrauterine red cell transfusion for parvovirus B19-induced fetal hydrops. BJOG 2002; 109: 1232-4.

[34] Donders GG, Van Lierde S, Van Elsacker-Niele AM, Moerman P, Goubau P, Vandenberge K. Survival after intrauterine parvovirus B19 infection with persistence in early infant: a two-year followup. Pediatr Infect Dis J 1994; 13: 234-6.

[35] Koch WC, Adler SP, Harger J. Intrauterine parvovirus B19 infection may cause an asymptomatic or recurrent postnatal infections. Pediatr Infect Dis J 1993; 12: 747-50.

[36] Nagel HT, de Haan TR, Vandenbussche FP, Opekes D, Walther FJ. Long-term outcome after fetal transfusion for hydrops associated with parvovirus B19 infection. Obstet Gynecol 2007; 109: 42-7.

[37] Perkin MA, English PM. Immediate and long term outcome of human parvovirus B19 infection in pregnancy. $\mathrm{Br} \mathrm{J}$ Obstet Gynaecol 1998; 105: 1337-8.

[38] Rodis JF, Rodner C, Hansen AA, Borgida AF, Deoliveira I, Rosengren SS. Long-term outcome of children following maternal human parvovirus B19 infection. Obstet Gynecol 1998; 91: 125-8.

[39] Sheikh AU, Ernest JM, O'Shea M. Long-term outcome in fetal hydrops from parvovirus B19 infection. Am J Obstet Gynecol 1992; 167: 337-41

[40] Wolke D, Söhne B, Ohrt B, Riegel K. Follow-up of preterm children: important to document dropouts. Lancet 1995; 345: 447.

[41] Kilham L, Margolis G. Problems of human concern arising from animal models of intrauterine and neonatal infections due to viruses: a review. I. Introduction and virologic studies. Prog Med Virol 1975; 20: 113-43.

[42] Margolis G, Kilham L. Problems of human concern arising from animal models of intrauterine and neonatal infections due to viruses: a review. II. Pathologic studies. Prog Med Virol 1975; 20: 144-79.

[43] Brown KE, Anderson SM, Young NS. Erythrocyte P antigen cellular receptor for B19 parvovirus. Science 1993; 262: 114-7.

[44] Ryan G, Kelly E, Inwood S, et al. Long term pediatric follow-up in non-immune hydrops secondary to parvovirus infection. Am J Obstet Gynecol 1997; 176: S86.

[45] Katz VL, McCoy MC, Kuller JA, Hansen WF. An association between fetal parvovirus B19 infection and fetal anomalies: a report of two cases. Am J Perinatol 1996; 13: 43-5.

[46] Isumi H, Nunoue T, Nishida A, Takashima S. Fetal brain infection with human parvovirus B19. Pediatr Neurol 1999; 21: 661-3.

[47] Barah F, Vallely PJ, Chiswick ML, Cleator GM, Kerr JR. Association of human parvovirus B19 infection with acute meningoencephalitis. Lancet 2001; 358: 729-30.

[48] Kerr JR, Barah F, Chiswick ML, et al. Evidence for the role of demyelination, HLA-DR alleles and cytokines in the pathogenesis of parvovirus B19 meningoencephalitis and its sequelae. J Neurol Neurosurg Psychiatry 2002; 73: 739-46.

[49] Doyle LW, Kelly EA, Rickards AL, Ford GW, Callanan C. Sensorineural outcome at 2 years for survivors of erythroblastosis treated with fetal intravascular transfusions. Obstet Gynecol 1993; 81: 931-5.

[50] Janssens HM, de Haan MJ, van Kamp IL, Brand R, Kanhai HH, Veen S. Outcome for children treated with fetal intravascular transfusions because of severe blood group antagonism. J Pediatr 1997; 131: 373-80

[51] Hudon L, Moise KJ, Hegemier SE, et al. Long-term neurodevelopmental outcome after intrauterine transfusion for the treatment of fetal hemolytic anemia. Am J Obstet Gynecol 1998; 179: 858-63.

[52] Crowcroft NS, Roth CE, Cohen BJ, Miller E. Guidance for control of parvovirus B19 infection in healthcare settings and the community. J Public Health Med 1999; 21: 439-46.

This is an open access article licensed under the terms of the Creative Commons Attribution Non-Commercial License (http://creativecommons.org/licenses/ by-nc/3.0/) which permits unrestricted, non-commercial use, distribution and reproduction in any medium, provided the work is properly cited. 